International Journal of Biological Sciences

ISSN 1449-2288 www.biolsci.org 2005 1(4):152-152

(C)2005 Ivyspring International Publisher. All rights reserved

Editorial

\title{
Mechanisms of Cellular Signal Transduction
}

\section{Shawn Li}

Department of Biochemistry, Schulich School of Medicine, University of Western Ontario, London, Ontario N6A 5C1, Canada

Corresponding address: Dr. Shawn Li. Telephone: (519) 850-2910; Fax: (519) 661-3175; email: sli@uwo.ca

Published: 2005.12.29

\section{Editorial}

Signal transduction is essential for almost all aspects of eukaryotic cell function and for the development of metazoans. In the classic case of signaling mediated by a growth factor, binding of the hormone to its receptor initiates a process that starts with the auto- or cross-phosphorylation of the receptor. The activating signal is then propagated through intracellular signaling pathways to the nucleus, resulting in alterations in gene expression. Understanding how extracellular stimuli and intracellular signals are transmitted in an effective and faithful manner inside a cell or between neighboring cells is vital to our understanding of such important biological processes as cell proliferation, differentiation, motility and survival. Our knowledge of how cells signal in normal and disease states has grown enormously in the past decade as many signaling pathways regulating a plethora of cellular behaviors and developmental events have been unraveled. In a previous issue of IJBS (vol 1, issue 2, pages 44-95), some of the most exciting recent developments that have taken place are reviewed by a panel of experts in the field.

The phosphoinositide 3-kinase (PI3K) is an important molecule downstream of growth factor signaling. Ahn and Ye (pages 44-50) discuss their recent finding that PI3K not only signals at the membrane-proximal region of the receptors, but has a key role in the nucleus. The activity of PI3K in the nucleus appears to be regulated by the PI 3-kinase enhancer (or PIKE), which in turn, is regulated by PLC- $\gamma 1$, acting as a physiologic guanine nucleotide exchange factor for PIKE. The heterotrimeric guanine nucleotide-binding proteins ( $G$ proteins) are known to transduce a variety of extracellular signals by coupling to the seven transmembrane domain (7TM) receptors. Siderovski and Willard (pages 51-66) expand from their early discovery of "regulator of G protein signaling" (RGS) proteins and provide a comprehensive and insightful account of the complexity of signaling events regulated by these $G$ proteins and associated molecules. Protein kinases play a central role in signaling processes essential for cell proliferation and survival, and are deregulated in various forms of cancers. Bibby and Litchfield (pages 67-79) offer an in-depth look at CK2 (formerly casein kinase II), a Ser/Thr kinase believed to be involved in transformation and tumorigenesis. They summarize biochemical and genetic evidence indicating that the regulatory subunit of CK2 possesses functions that are independent of the holoenzyme. Signal transduction also underlies tissue remodeling and animal development. For instance, the maintenance and regeneration of the epidermis is centrally controlled by the E2F transcription factor regulated network. On pages 87-95, Ivanova et. al provide an update on the functions and mechanisms of action for the various components of the E2F/pRb growth regulatory pathway. Finally, in a lucid and convincing manner, Rao (pages 80-86) discusses the importance of the NCK/Dock signaling pathways in axonal guidance and neuronal targeting and how the Drosophila visual system offers a powerful tool by which to dissect the components of signaling pathways coordinated by this important adaptor protein.

With the rapid growth in signal transduction in recent years, the literature has become so vast that it is virtually impossible to include all relevant areas in a single issue. The special issue is therefore assembled to give our readers a glimpse of specific topics within this exciting field. We welcome suggestions for future topics and submissions of reviews that provide timely coverage of significant development in this and other areas of biomedical research.

\section{Conflict of interest}

The author has declared that no conflict of interest exists. 